\title{
Hemodynamic Responses to Speech and Music in Newborn Infants
}

\author{
Kalle Kotilahti, ,2* Ilkka Nissilä,' Tiina Näsi, ,,2 Lauri Lipiäinen,' \\ Tommi Noponen, ',3 Pekka Meriläinen,' Minna Huotilainen, ${ }^{4,5}$ \\ and Vineta Fellman ${ }^{6,7}$ \\ ${ }^{1}$ Department of Biomedical Engineering and Computational Science, Helsinki University of \\ Technology, Espoo, Finland \\ ${ }^{2}$ BioMag Laboratory, HUSLAB, Hospital District of Helsinki and Uusimaa, \\ Helsinki University Central Hospital, Helsinki, Finland \\ ${ }^{3}$ Turku PET Centre, Turku University Hospital, Turku, Finland \\ ${ }^{4}$ Cognitive Brain Research Unit, Department of Psychology, University of Helsinki, Helsinki, Finland \\ ${ }^{5}$ Finnish Centre of Excellence in Interdisciplinary Music Research, Department of Music, University of \\ Jyväskylä, Jyväskylä, Finland \\ ${ }^{6}$ Department of Pediatrics, University of Helsinki, Helsinki, Finland \\ ${ }^{7}$ Department of Pediatrics, Lund University, Lund, Sweden
}

\begin{abstract}
We used near-infrared spectroscopy (NIRS) to study responses to speech and music on the auditory cortices of 13 healthy full-term newborn infants during natural sleep. The purpose of the study was to investigate the lateralization of speech and music responses at this stage of development. NIRS data was recorded from eight positions on both hemispheres simultaneously with electroencephalography, electrooculography, electrocardiography, pulse oximetry, and inclinometry. In 11 subjects, statistically significant $(P$ $<0.02)$ oxygenated $\left(\mathrm{HbO}_{2}\right)$ and total hemoglobin $(\mathrm{HbT})$ responses were recorded. Both stimulus types elicited significant $\mathrm{HbO}_{2}$ and $\mathrm{HbT}$ responses on both hemispheres in five subjects. Six of the 11 subjects had positive $\mathrm{HbO}_{2}$ and $\mathrm{HbT}$ responses to both stimulus types, whereas one subject had negative responses. Mixed positive and negative responses were observed in four neonates. On both hemispheres, speech and music responses were significantly correlated $(r=0.64 ; P=0.018$ on the left hemisphere (LH) and $r=0.60$; $P=0.029$ on the right hemisphere $(\mathrm{RH})$ ). On the group level, the average response to the speech stimuli was statistically significantly greater than zero in the LH, whereas responses on the RH or to the music stimuli did not differ significantly from zero. This suggests a more coherent response to speech on the LH. However, significant differences in lateralization of the responses or mean response amplitudes of the two stimulus types were not observed on the group level. Hum Brain Mapp 31:595-603, 2010.
\end{abstract}

Key words: near-infrared spectroscopy; functional laterality; auditory responses; neonate; brain imaging

Contract grant sponsor: Academy of Finland; Contract grant number: 120946.

Contract grant sponsors: Instrumentarium Science Foundation, the Finnish Cultural Foundation, Helsinki University of Technology Research Training Scholarships, Jenny and Antti Wihuri Foundation.

*Correspondence to: Kalle Kotilahti, Department of Biomedical Engineering and Computational Science, Helsinki University of
Technology, P.O. Box 3310, FIN-02015 TKK, Finland. E-mail: kalle. kotilahti@tkk.fi

Received for publication 5 February 2009; Revised 17 July 2009; Accepted 24 July 2009

DOI: $10.1002 / \mathrm{hbm} .20890$

Published online 29 September 2009 in Wiley InterScience (www. interscience.wiley.com).

(C) 2009 Wiley-Liss, Inc. 


\section{INTRODUCTION}

Near-infrared spectroscopic imaging (NIRSI) is a noninvasive technique for measuring the concentrations of oxygenated and deoxygenated hemoglobin $\left(\mathrm{HbO}_{2}\right.$ and $\left.\mathrm{HbR}\right)$ in tissue. An interesting application of the method is the study of hemodynamic responses to brain activation. NIRSI measures local changes in the absorption of nearinfrared light which are affected by changes in the cerebral blood volume (CBV) and oxygenation $\left(\mathrm{StO}_{2}\right)$. It is completely noninvasive and does not use ionizing radiation. Further, it does not interfere electromagnetically with other neuroimaging modalities or require a shielded environment. Thus, NIRSI can be performed during electroencephalography (EEG), magnetoencephalography (MEG), functional magnetic resonance imaging, or positron emission tomography without causing artifacts in the recordings. As NIRSI can be implemented in such a way that bedside measurements are possible, it is an attractive method for imaging and monitoring of newborn infants being safe, portable, and silent.

NIRS has been applied to activation studies of pre- and full-term newborns, e.g., in the auditory [e.g., Chen et al., 2002; Kotilahti et al., 2005; Nishida et al., 2008; Peña et al., 2003; Zaramella et al., 2001], visual [e.g. Karen et al., 2008; Kusaka et al., 2004; Taga et al., 2003], passive motor [e.g., Hintz et al., 2001; Isobe et al., 2001, Gibson et al., 2006], olfactory [Bartocci et al., 2000, 2001], and pain [Bartocci et al., 2006] modalities. The method is undergoing rapid development [see e.g., Zeff et al., 2007 for an adult visual mapping study) and more advanced implementations in the future are likely to have localization accuracy of 2$3 \mathrm{~mm}$ in the parts of the cortex closest to the skin in neonates [Heiskala et al., 2009]. With these advancements, the use of NIRS in clinical practice as well as pediatric neuroscience is likely to increase rapidly in the near future. New 2-4 channel NIRS equipment are already commercially available and introduced for monitoring of tissue oxygenation during neonatal intensive care. Thus, it is important to assess responses in healthy newborns to understand the basic physiology of NIRS responses to relevant stimuli, such as naturally occurring sounds.

Processing of auditory information like speech and music is facilitated by the auditory system already at birth compared to sounds like sinusoidal tones, which are not specific to human communication and typically give smaller cortical responses than speech and music sounds [Huotilainen, 2006]. Brain research methods like NIRSI and MEG, which measure cortical activity without remarkable contribution from lower brain areas, are interesting for the study of the developing cortex because measurements with these methods highlight the functions of the still-developing cortical regions in the light of the incoming information from the lower brain areas. These methods, when used in the newborn period, can give insight into the specialization of the auditory cortical areas, seen in adults as robust speech-sound-specific areas in the left and melody-harmony-specialized areas in the right temporal lobe.

In adults, the key acoustic characteristics of speech, i.e., very fast transitions of spectral peak frequencies or formants, short periods of noise with varying characteristics, short periods of silence, etc., give rise to a lot of activity in the left temporal areas, while the key acoustic characteristics of melodic and harmonic music, i.e., the matching of overtones of simultaneously presented fundamental pitches, continuation of pitch levels across the whole musical piece, and long-lasting timbral qualities of sound, activate the right auditory areas [e.g., Tervaniemi and Hugdahl, 2003]. The development of this specialization during ontogenesis is still somewhat unclear. It has been suggested that the left hemisphere (LH) dominates all sound processing in neonates [Bradshaw and Nettleton, 1983], and that neonates already have specific structures for processing speech-like sounds in the LH [Hellige, 1993; Mehler and Dupoux, 1994], which would lead to stronger responses to speech on the $\mathrm{LH}$.

Several studies have investigated characteristics of the lateralization of speech perception and whether the $\mathrm{LH}$ dominance of language processing is already present in neonatal brain or if it is developed during the early infancy. Peña et al. [2003] found significant responses to speech only in the LH in newborns. On the other hand, Taga and Asakawa [2007] did not observe lateralized patterns of activation in response to speech sounds in fully attentive infants aged 2-4 months, and Homae et al. [2006] found stronger responses on right hemisphere (RH) to speech with pitch information in 3-month-old infants. However, the fMRI study by Dehaene-Lambertz et al. [2002] shows adult-like lateralization of responses to speech sounds in 3-months-old infants. Further study is, however, needed to clarify the issue especially in newborn brain as most of the previous studies have been done with 2- to 4-month-old infants.

Our aim was to study the asymmetries in the processing of natural speech in the cortical areas of newborn infants' left and right temporal lobes using music as a counterpart to the speech stimuli. The measurements were carried out using NIRSI during natural sleep.

\section{MATERIALS AND METHODS}

\section{Participants}

We studied 13 (9 male) healthy full-term newborn infants (gestational age from 37.3 to 42.3 weeks, mean 39.6 $\pm 1.2 \mathrm{SD}$ ) during natural sleep. The ages of the infants were from 1 to 4 postnatal days (mean $1.8 \pm 1.0 \mathrm{SD}$ ). The study was approved by the Ethics Committee for Pediatrics, Adolescent Medicine and Psychiatry, Hospital District of Helsinki and Uusimaa and it is in compliance with the Declaration of Helsinki. Written informed consent was obtained from a parent before the subject was enrolled in the study. 


\section{Study Design}

Auditory stimuli used in this study consisted of 5-s samples of speech and music. The speech samples were extracted from the fairy tale "Little red riding hood" read in Finnish by a Finnish actress using infant-directed intonation with high variations in the intensity, pitch, and temporal structure. The music samples were extracted from a piano concerto by Mozart. The stimuli were presented via loudspeakers placed at a distance of $1.5 \mathrm{~m}$ from the infant's head, and with a sound intensity of approximately $60 \mathrm{~dB}$ at the level of the infant's head. When compared across the 5-s intervals, the speech and music samples contained approximately similar frequency characteristics.

Stimuli were ordered randomly using an optimal eventrelated paradigm [Dale, 1999], so that each stimulus was followed by a silent rest period of varying length (mean $15 \mathrm{~s}$ ). Samples of both stimulus types were presented up to 64 times during an experiment. The stimuli were controlled with Presentation ${ }^{\circledR}$ software (Version 9.30, www.neurobs.com).

Measurements were carried out in a quiet, dimly lit room while the neonate was sleeping in the supine position in the lap of a nurse. The neonatal sleep can roughly be divided into two sleep stages, active sleep (AS) and quiet sleep (QS), which are considered to be precursors of adult sleep stages. AS resembles the adult REM sleep and is somewhat restless, whereas QS is deeper and more restful. The arousal stages (awake, AS, and QS) were determined by the nurse during the measurement based on muscular tension, breathing, eye movements, and behavioral movements of the infant. The experiment was discontinued if the infant woke up.

\section{Instrumentation}

The NIRSI study was carried out using a 16-channel frequency-domain (FD) instrument [Nissilä et al., 2002, 2005] developed at Helsinki University of Technology. The measurements were performed simultaneously above both auditory cortices on the temporal lobes. From each of the two auditory areas, data were collected from eight locations (Fig. 1a) with two source-detector distances (SDDs) of 15 and $25 \mathrm{~mm}$.

The data were recorded using light from two laser diodes modulated at $100 \mathrm{MHz}$ with wavelengths of 692 and $832 \mathrm{~nm}$. Optical fibers were used to deliver the nearinfrared light to the skin surface and from the skin to the detectors. An adjustable and adaptable fiber-coupling helmet was applied to ensure a safe and comfortable interface between the instrument and the infant (Fig. 1b). The amplitude and the phase of the modulation of the measured optical signals were calculated with a lock-in amplifier and sampled at approximately $12.5 \mathrm{~Hz}$.

The amplitude and phase values were calibrated before further data analysis using a three-step procedure that is explained in detail in Tarvainen et al. [2005] and Nissilä et al. [2005]. The first step is to determine the effects of de- (a)
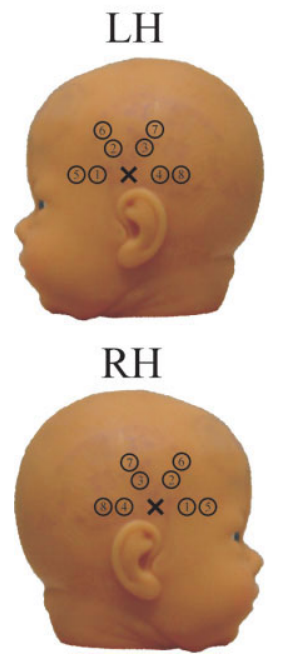

(a) Source (cross) and detector (circle) positions on the left and RH. (b) The measurement setup. Black optical fibers and fiber bundles are attached on top of the auditory cortices. The inclinometer is visible on top of the forehead. [Color figure can be viewed in the online issue, which is available at www. interscience.wiley.com.]

tector gain adjustment on the measured amplitude and phase of the output of each detector. The second step determines the differences in attenuation and phase shift because of differences in the physical lengths and contacts in each channel of the instrument. The third step of calibration involves the measurement of a reference object with one source and one detector channel, providing the zero point of phase. The calibration procedure is carried out for each wavelength used in the study.

The optical power on the surface of the tissue was at most $5 \mathrm{~mW}$, which is enough to exceed the maximum permissible exposure (MPE) for eyes if the beam is collimated (IEC 60825 standard). However, the light coming out of an optical fiber such as used in this study is not collimated and the geometry of the fiber-coupling helmet was such that the MPE could not have been exceeded on subject's eyes at any time. In addition, the instrumentation was built in such a way that the laser radiation is shut down automatically if the fiber-coupling helmet is detached. The light intensity on the brain surface during the measurement was approximately $5 \%$ of that under midday sunlight on a sunny day in midsummer [Kiguchi et al., 2007].

Electrooculography (EOG), electrocardiography, and four channels of scalp EEG, respiratory impedance, arterial oxygen saturation and subject movements were recorded simultaneously during the NIRSI measurements with an anesthesia monitor (AS/3 Anesthesia Monitor, DatexOhmeda, Finland). The respiratory impedance was measured between EEG-reference and ECG electrodes. The 
arterial oxygen saturation was measured with a pulse oximetry sensor (FlexSat, Datex-Ohmeda, Finland), which was attached to the right foot of the infant. The movements of the infant were monitored using an inclinometer attached on the forehead (Fig. 1b).

\section{Data Analysis}

To reduce the effect of drift and slow oscillations due to background physiology and optode contact variation, the signals were de-trended by dividing them with the lowpass filtered $(-3 \mathrm{~dB}$ cutoff frequency at $0.02 \mathrm{~Hz}$ ) versions. After de-trending, the amplitude signal was lowpass filtered $(-3 \mathrm{~dB}$ cutoff frequency at $0.5 \mathrm{~Hz}$ ) to reduce highfrequency oscillations such as those induced by the pulsation of the heart. After filtering, the signals were downsampled to a frequency of $1 \mathrm{~Hz}$.

Optical path lengths were calculated for all source-detector pairs for the nine subjects with successfully calibrated phase measurements. The mean differential path length factors (DPFs) for both wavelengths and SDDs were calculated by dividing the optical path lengths with the corresponding SDDs. DPFs averaged over subjects with calibrated phase values are shown in Table I.

To minimize the influence of external light from the room, changes in the modulation amplitude of the optical signal were used in place of changes in the attenuation of the light in the tissue in the analysis. The amplitudes were transformed into concentration changes of oxygenated, deoxygenated, and total hemoglobin $\left(\mathrm{HbO}_{2}, \mathrm{HbR}\right.$, and $\mathrm{HbT}$, respectively) using the modified Beer-Lambert law. The measured optical path lengths were used in the calculation of modified Beer-Lambert law for the nine infants with calibrated phase values. For the four neonates without calibrated phase values, the mean DPF values measured in this study were used (Table I). Head movements were detected from the inclinometer data and used to reject motion artefacts from the NIRSI data. In addition, an individually selected peak-to-peak threshold between 7 and $18 \mu \mathrm{M}$ was used to reject those stimulation epochs that contained abrupt changes or spikes in the amplitude signal as they were most likely to be caused by motion artefacts or contact changes.

The statistical analysis of the hemodynamic responses was based on the general linear model (GLM), which models $\mathrm{HbO}_{2}, \mathrm{HbR}$, and $\mathrm{HbT}$ concentration time series based on the stimulus timings and a hemodynamic response function (HRF) [e.g., Kiebel et al., 2003]. The predictor for the HRFs was a two parameter gamma-based function. The first temporal derivative of the HRF was included to allow a change in the latency of the response [e.g., Smith, 2004]. The predictor was convolved with stimulus vectors (a delta function indicating the onset of the stimulus) and fitted to the concentration time series in the least-squares sense. The GLM produced beta parameters which represent the area-under-the-curve (AUC) of the
TABLE I. Differential path length factors (mean \pm SE)

for both source-detector distances and wavelengths averaged over nine neonates and all source-detector pairs

\begin{tabular}{lcc}
\hline & \multicolumn{2}{c}{ Wavelength } \\
\cline { 2 - 3 } & $692 \mathrm{~nm}$ & $832 \mathrm{~nm}$ \\
\hline Source-detector distance & & \\
$15 \mathrm{~mm}$ & $4.35 \pm 0.16$ & $3.64 \pm 0.19$ \\
$25 \mathrm{~mm}$ & $4.56 \pm 0.17$ & $3.96 \pm 0.14$ \\
\hline
\end{tabular}

HRFs corresponding to the responses evoked by the different stimuli (the AUC of the predictor of the HRF was scaled to unity).

The AUCs of the $\mathrm{HbO}_{2}, \mathrm{HbR}$, and $\mathrm{HbT}$ concentration changes between 0 and 20 s with respect to the stimulus onset were considered as the magnitudes of the corresponding hemodynamic response and tested for statistical significance with a two-tailed Student's $t$-test. The multiple comparison problem was considered by calculating the Dubey/Armitage-Parmar correction [e.g., Sankoh et al., 1997], which takes into account the spatial correlation between the measurement channels. The mean spatial correlations between channels on a hemisphere were 0.72 for $\mathrm{HbO}_{2}, 0.59$ for $\mathrm{HbR}$, and 0.69 for HbT. Thus, the significance level of 0.05 was corrected to 0.02 for $\mathrm{HbO}_{2}, 0.03$ for $\mathrm{HbR}$, and 0.02 for $\mathrm{HbT}$.

Maximal $\mathrm{HbO}_{2}, \mathrm{HbR}$, and $\mathrm{HbT}$ amplitude and AUC values (the channel with the largest response was chosen here), as well as the time from stimulus onset to the maximal value (time-to-peak (TTP) of the fitted response) were also determined for hemodynamic responses with magnitudes that differ significantly from zero.

The lateralization of the responses was studied by calculating the laterality index LI $=(L-R) /(L+R)$ [Minagawa-Kawai et al., 2007], where $L$ is the maximal $\mathrm{HbT}$ AUC on the $\mathrm{LH}$ and $R$ is the maximal $\mathrm{HbT}$ AUC on the RH. Thus, an LI value below zero represents righthemisphere dominance and LI above zero left-hemisphere dominance.

\section{RESULTS}

Statistically significant $(P<0.02)$ hemodynamic responses (HbT AUC) were recorded in 11 infants. Five of the infants had statistically significant $\mathrm{HbT}$ responses for both stimulus conditions on both hemispheres.

The $\mathrm{HbO}_{2}$ and $\mathrm{HbT}$ concentrations increased in response to auditory stimulation ("positive responses") in six neonates. In one neonate, however, $\mathrm{HbO}_{2}$ and $\mathrm{HbT}$ concentrations decreased significantly in response to both stimuli ("negative responses"). Four neonates had mixed responses (positive and negative $\mathrm{HbO}_{2}$ and $\mathrm{HbT}$ responses depending on the hemisphere and the stimulus condition). Maximal $\mathrm{HbT}$ response AUCs for both stimuli and 
- Neonatal Responses to Speech and Music •

TABLE II. Individual maximal HbT AUCs $(\mu M s)$ for neonates with significant responses $(* P<0.02$ and $* * P<0.00 \mathrm{I})$, average $\mathrm{HbT} A \mathrm{AUCs}$ and total numbers of responses over neonates with significant responses for left and right hemispheres ( $\mathrm{LH}$ and $\mathrm{RH}$ ) and for speech and music stimuli (S and $\mathrm{M}$ )

\begin{tabular}{|c|c|c|c|c|c|c|}
\hline \multirow[b]{2}{*}{ Subject } & \multicolumn{3}{|c|}{ LH } & \multicolumn{3}{|c|}{ RH } \\
\hline & $S$ & M & S-M & S & M & S-M \\
\hline 1 & $3.27^{*}$ & $-4.57^{*}$ & $7.84^{* *}$ & $7.10^{* *}$ & $-4.29 *$ & $11.39^{* *}$ \\
\hline \multicolumn{7}{|l|}{2} \\
\hline 3 & $6.78^{* * *}$ & $-4.42^{*}$ & $11.20^{* *}$ & $-3.93^{*}$ & $-10.30^{* * *}$ & $6.37^{*}$ \\
\hline 4 & $6.27^{*}$ & & & $8.43^{*}$ & $8.00^{*}$ & \\
\hline 5 & $4.54^{*}$ & $6.06^{*}$ & & $10.81^{*}$ & $5.09^{*}$ & $5.72^{*}$ \\
\hline 6 & $16.81^{*}$ & $20.80^{*}$ & & & $12.42^{*}$ & $-13.69^{*}$ \\
\hline 7 & $5.64^{*}$ & $10.94^{*}$ & $-5.30^{*}$ & $14.43^{* *}$ & $19.57^{* *}$ & $-5.14^{*}$ \\
\hline 8 & $1.30^{*}$ & $7.59^{*}$ & $-6.29 *$ & $5.81^{* *}$ & $7.61^{*}$ & \\
\hline 9 & & $-6.92^{*}$ & & $10.47^{*}$ & & $6.92^{*}$ \\
\hline 10 & & & & $7.64^{*}$ & & $4.26^{*}$ \\
\hline 11 & $-1.62^{*}$ & & & $-2.49^{*}$ & & \\
\hline \multicolumn{7}{|l|}{12} \\
\hline 13 & $3.23^{*}$ & & & $-5.83^{*}$ & & $-4.13^{*}$ \\
\hline Average & $4.27^{*}$ & 3.30 & & 4.25 & 3.38 & \\
\hline Numbers of neonates with & 9 & 7 & 4 & \multirow{2}{*}{\multicolumn{2}{|c|}{6}} & 8 \\
\hline significant responses & \multicolumn{2}{|c|}{6} & & & & \\
\hline
\end{tabular}

The difference between the maximal speech and music responses (S-M) is shown for each subject, if it is significant in two-tailed Student's $t$-test.

hemispheres for all subjects, and the numbers of subjects showing significant responses to each stimulus type and on each hemisphere are shown in Table II and illustrated in Figure 2.

Pearson's correlation coefficient between the maximal music and speech responses (HbT AUC of the channel giving the largest response magnitude) in all neonates was $0.64(P=0.018)$ on $\mathrm{LH}$ and $0.60(P=0.029)$ on RH. In the neonates with consistent uniform responses (only positive or only negative responses for both conditions), the corresponding coefficients were $0.89(P=0.0011, N=9)$ and $0.84(P=0.0012, N=11)$.

For statistically significant positive $\left(\mathrm{HbO}_{2}\right.$ and $\left.\mathrm{HbT}\right)$ responses, the mean peak for $\mathrm{HbO} 2, \mathrm{HbR}$, and $\mathrm{HbT}$ concentration changes were $0.81 \pm 0.08 \mu \mathrm{M},-0.29 \pm 0.04 \mu \mathrm{M}$, and $0.89 \pm 0.10 \mu \mathrm{M}$ (mean $\pm \mathrm{SE}$ ) respectively and their latencies were $13.2 \pm 0.6 \mathrm{~s}, 12.3 \pm 0.6 \mathrm{~s}$, and $13.2 \pm 0.5 \mathrm{~s}$ (mean \pm SE) with respect to stimulus onset. See Table III for more detailed information on the average values regarding hemispheres and stimulus conditions. Time courses of hemodynamic responses $\left(\mathrm{HbO}_{2}\right.$ and $\left.\mathrm{HbR}\right)$ averaged for both hemispheres over neonates and measurement channels with significant responses are presented in Figure 3. The response magnitudes (HbT AUCs) averaged over all the subjects with significant positive responses are presented in Figure 4.

Locations of maximal significant $\mathrm{HbT}$ AUCs (from the channel giving the largest response) are presented in Figure 5. On the $\mathrm{RH}$, more significant responses were detected with the short SDD than with the long SDD. On the $\mathrm{LH}$, numbers of significant responses were equal for
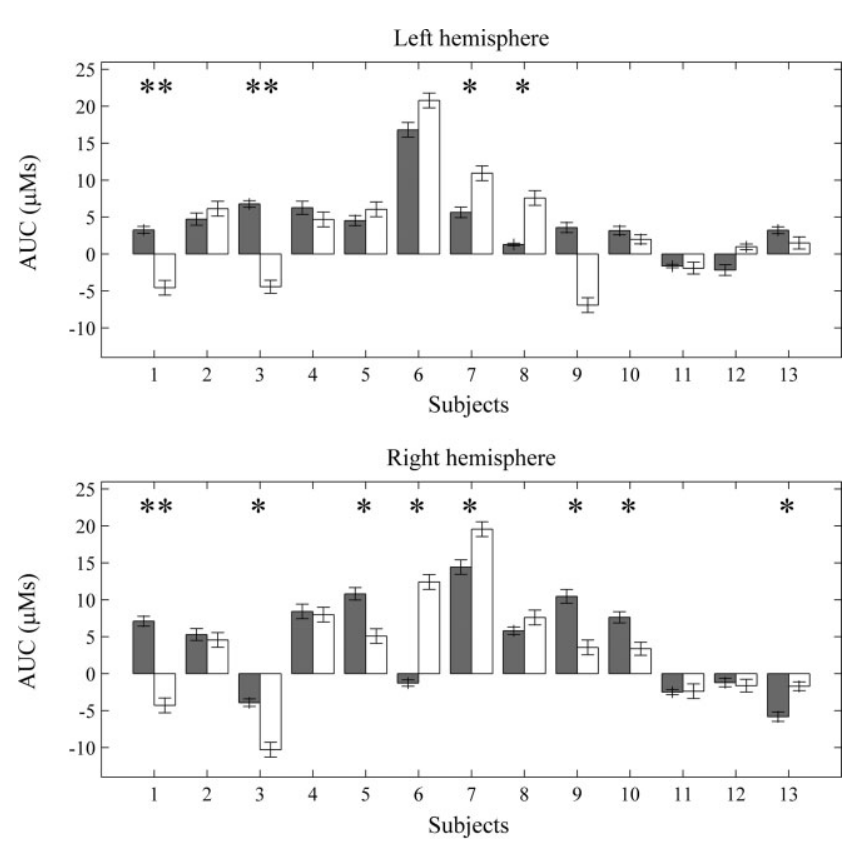

Figure 2.

Maximal speech (gray) and music (white) HbT AUCs on the LH and $\mathrm{RH}$ for all $\mathrm{I} 3$ neonates. The error bars indicate the standard error of the AUC. Subjects with a significant difference between the stimulus conditions are marked with asterisks ( $* P<0.02$ and $* * P<0.001$ ). 
TABLE III. Mean maximal $\mathrm{HbO}_{2}, \mathrm{HbR}$, and $\mathrm{HbT}$ peak values, TTPs and AUCs (mean \pm SE) for neonates with positive significant responses $\left(P<0.02 \mathrm{HbO}_{2}\right.$ and $\mathrm{HbT}, P<0.03$ for $\left.\mathrm{HbR}\right)$

\begin{tabular}{|c|c|c|c|c|c|c|c|}
\hline & \multirow[b]{2}{*}{ Stim. } & \multicolumn{2}{|c|}{ Peak value $(\mu \mathrm{M})$} & \multicolumn{2}{|c|}{ Time-to-peak (s) } & \multicolumn{2}{|c|}{ AUC ( $\mu \mathrm{Ms})$} \\
\hline & & $\mathrm{LH}$ & $\mathrm{RH}$ & LH & $\mathrm{RH}$ & LH & $\mathrm{RH}$ \\
\hline \multirow[t]{2}{*}{$\mathrm{HbO}_{2}$} & $S$ & $0.63 \pm 0.10$ & $0.88 \pm 0.11$ & $12.2 \pm 1.1$ & $13.1 \pm 1.1$ & $5.04 \pm 0.94$ & $7.66 \pm 1.22$ \\
\hline & M & $1.00 \pm 0.25$ & $0.89 \pm 0.27$ & $14.1 \pm 0.6$ & $14.3 \pm 1.3$ & $10.33 \pm 1.11$ & $9.22 \pm 1.88$ \\
\hline \multirow[t]{2}{*}{$\mathrm{HbR}$} & $S$ & $-0.22 \pm 0.05$ & $-0.25 \pm 0.05$ & $11.8 \pm 1.1$ & $11.0 \pm 1.6$ & $-1.88 \pm 0.52$ & $-2.32 \pm 0.52$ \\
\hline & M & $-0.43 \pm 0.10$ & $-0.39 \pm 0.10$ & $12.9 \pm 1.0$ & $13.5 \pm 1.0$ & $-3.49 \pm 0.74$ & $-3.94 \pm 1.07$ \\
\hline \multirow[t]{2}{*}{$\mathrm{HbT}$} & $S$ & $0.62 \pm 0.16$ & $0.99 \pm 0.09$ & $11.9 \pm 1.0$ & $12.9 \pm 1.1$ & $5.46 \pm 1.37$ & $9.24 \pm 1.10$ \\
\hline & M & $1.11 \pm 0.32$ & $1.01 \pm 0.24$ & $13.8 \pm 0.5$ & $15.1 \pm 1.1$ & $11.35 \pm 3.31$ & $9.34 \pm 2.40$ \\
\hline
\end{tabular}

both SDDs. Also, significant responses were found to be more posterior on the LH than on the RH.

For neonates with significant responses $(N=11)$, the laterality index was $-0.15 \pm 1.70$ (mean \pm SD) for speech and $-1.25 \pm 4.99$ for music. The LIs did not statistically significantly differ from zero or from each other in Student's $t$-test.

\section{DISCUSSION}

No statistically significant lateralization was found using LIs of speech and music responses as criteria. This result is in line with the results by Minagawa-Kawai et al. [2007], who observed a significant leftward lateralization of phonemic-specific NIRS responses only in infants $>1$ year of age.

When considering the results on a group level, however, the speech response in the $\mathrm{LH}$ was the only statistically significant response (Table II). This finding may suggest a stronger or more uniform involvement of the LH for the speech stimulus in general in the newborn period. This is in line with the findings of Pena et al. [2003] and supports the theory that neonates have specific structures for processing speech-like sounds in the LH [Mehler and Dupoux, 1994].

The explanation for the negative responses to the stimuli used, i.e., decrease of $\mathrm{HbO}_{2}$ and $\mathrm{HbT}$ concentrations during and after sound stimulation might be a reduced activity in the probed area [Raichle and Mintun, 2006]. However, this is unlikely since the auditory areas are "hard-wired" and receive little activity from the lower brain areas. A more plausible explanation is the so-called blood stealing effect, in which the areas surrounding the activated area receive reduced blood flow compared to baseline. If this is the case, the negative responses may be a result of activity deeper in the brain or in areas that were close to but not inside the area measured with NIRSI. Thus, strong activity in the auditory cortex would cause

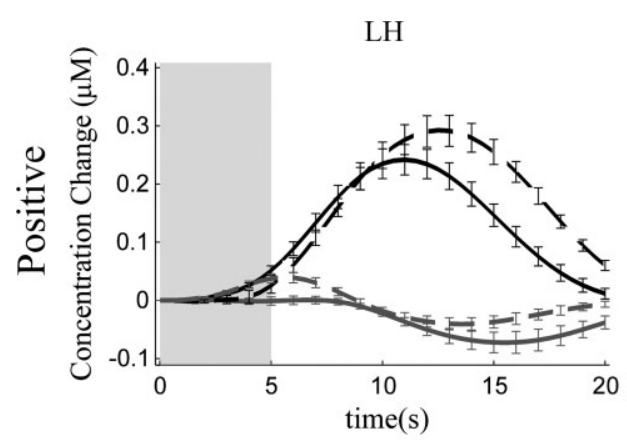

LH

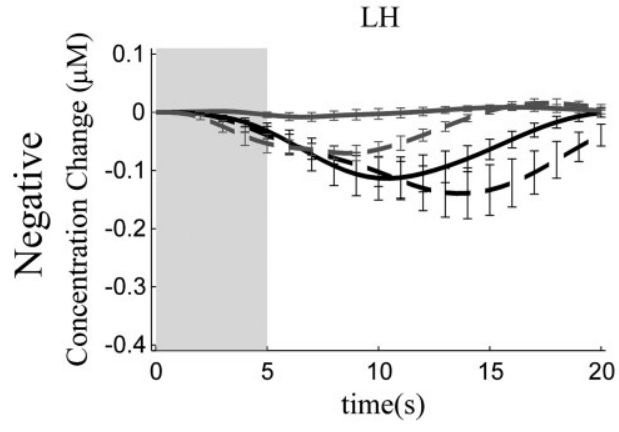

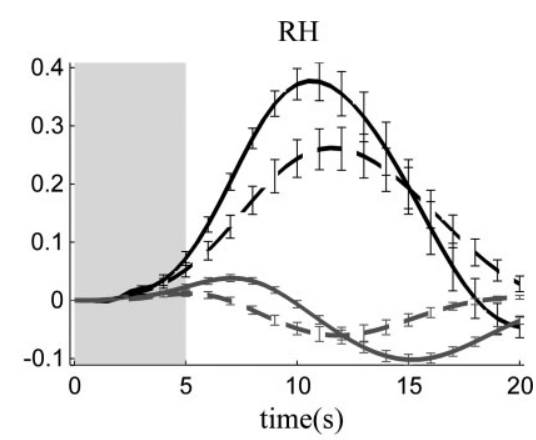

RH

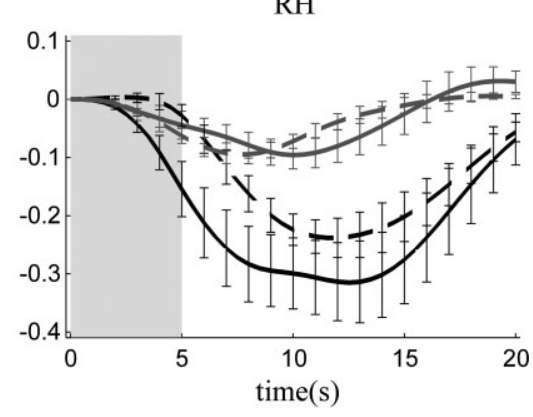

Figure 3.

Time courses of the oxygenated (black) and deoxygenated (gray) hemoglobin concentration changes relative to the stimuli (speech, solid line; music, dashed line) averaged over neonates with significant $(P<0.02 / 0.03)$ positive and negative responses. The gray rectangle indicates the time period when the stimulus was presented and the errorbars correspond to the standard error of the mean. 
an increase in $\mathrm{HbO}_{2}$ and $\mathrm{HbT}$ in the auditory cortex and a smaller decrease in $\mathrm{HbO}_{2}$ and $\mathrm{HbT}$ concentrations in the neighboring areas. It has been suggested that the regulation of CBF is still under development in neonates, which may partly explain the frequent occurrence of "negative" responses in studies of the neonate.

We found that the speech and music stimuli, equalized in duration and frequency content, elicited clear and significant responses in healthy neonates. In five neonates of 13 responses for speech stimulus were stronger in both hemispheres, whereas in three neonates responses for music stimulus were stronger in both hemispheres (Table II). There was no significant difference between the mean response amplitudes of the two stimulus types at group level (Table III), which suggests that speech and music elicit approximately the same amount of brain activity in the newborn period.

Maximal significant responses were found to be more posterior on the LH than on the RH. This is consistent with auditory MEG responses in adults, which are about $10 \mathrm{~mm}$ more anterior in the right than LH [Elberling et al., 1982; Nakasato et al., 1995]. This suggests that NIRSI can yield useful spatial information in functional measurements.

No significant differences were seen between responses measured in active and quiet sleep, although such differences have been previously reported using simpler sinusoidal beep stimuli [Kotilahti et al., 2005]. It is possible that the large intersubject variation in responses to speech and music, and variation in responses between individual stimuli hide differences in responses between sleep stages.

This study demonstrates high variability in brain responses between newborn infants using simple nonquantitative NIRS measurements. A part of the variability may be due to anatomical and physiological differences between hemispheres and individuals. Quantitative reconstructions of hemoglobin concentration changes using a
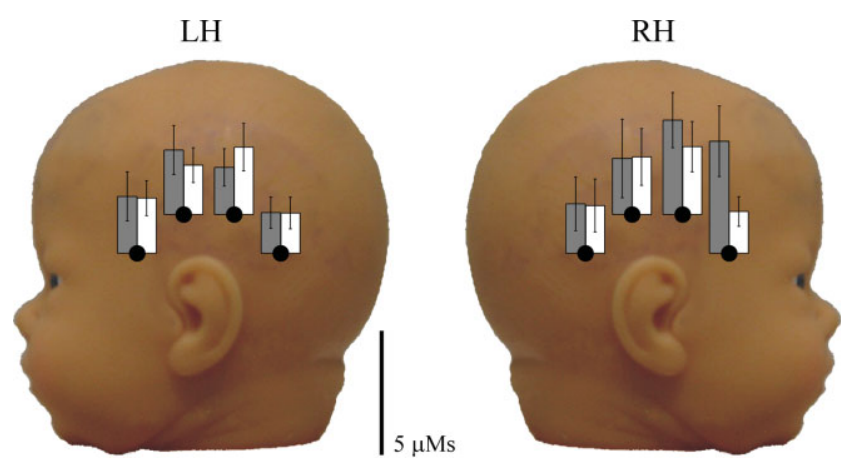

Figure 4.

The HbT AUCs for speech (gray) and music (white) responses averaged over the subjects with significant $(P<0.02)$ positive responses (eight subjects). Values are shown for only the long SDD. The errorbars indicate the standard deviations. [Color figure can be viewed in the online issue, which is available at www.interscience.wiley.com.]

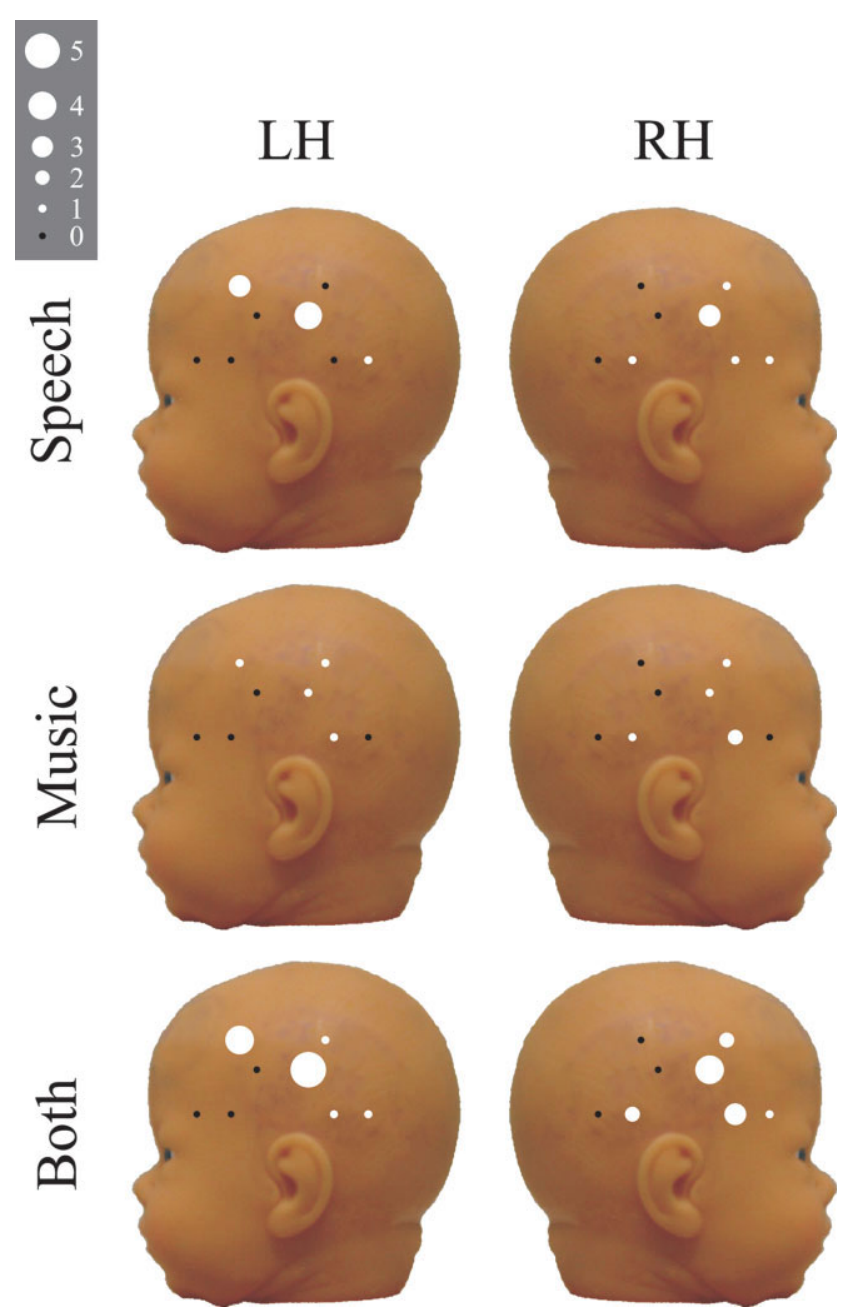

Figure 5.

Variations in the locations of maximal significant HbT AUCs over all neonates for each detector location on both hemispheres for each stimulus condition separately and both conditions summed. The diameter of the marker indicates number of maximal responses at that measurement site. [Color figure can be viewed in the online issue, which is available at www. interscience.wiley.com.]

dense optode grid and a model based reconstruction algorithm with anatomical a priori information [e.g., Heiskala et al., 2009] is likely to allow more sensitive lateralisation studies.

\section{CONCLUSION}

In this study, no significant consistent lateralization of speech or music responses appeared at group level. Both hemispheres could be activated by speech as well as by music stimuli, consistent with findings on adults [Tervaniemi and Hugdahl, 2003]. The responses were not significant in all newborns for both stimuli on both 
hemispheres, suggesting individual variations in hemodynamic responses in healthy newborns.

The significant group level responses to speech stimulus on LH suggests that newborns may have stronger or more developed speech and language related brain activation in the LH compared to the $\mathrm{RH}$, or compared to music related activation. This suggests that the typical speech lateralization to the LH has already started in the newborn, but it is not yet fully developed.

\section{ACKNOWLEDGMENT}

We are grateful to R.N. Leena Wallendahr for her assistance in the measurements.

\section{REFERENCES}

Bartocci M, Winberg J, Ruggiero C, Bergqvist LL, Serra G, Lagercrantz H (2000): Activation of olfactory cortex in newborn infants after odor stimulation: A functional near-infrared spectroscopy study. Pediatr Res 48:18-23.

Bartocci M, Winberg J, Papendieck G, Mustica T, Serra G, Lagercrantz H (2001): Cerebral hemodynamic response to unpleasant odors in the preterm newborn measured by near-infrared spectroscopy. Pediatr Res 50:324-330.

Bartocci M, Bergqvist LL, Lagercrantz H, Anand KJ (2006): Pain activates cortical areas in the preterm newborn brain. Pain 122:109-117.

Bradshaw JL, Nettleton N (1983): Human cerebral asymmetry. Englewood Cliffs, NJ: Prentice-Hall. 335 p.

Chen S, Sakatani K, Lichty W, Ning P, Zhao S, Zuo H (2002): Auditory-evoked cerebral oxygenation changes in hypoxic-ischemic encephalopathy of newborn infants monitored by near infrared spectroscopy. Early Hum Dev 67:113-121.

Dale AM (1999): Optimal experimental design for event-related fMRI. Hum Brain Mapp 8:109-114.

Dehaene-Lambertz G, Dehaene S, Hertz-Pannier L (2002): Functional neuroimaging of speech perception in infants. Science 298:2013-2015.

Elberling C, Bak C, Kofoed B, Lebech J, Saermark K (1982): Auditory magnetic fields from the human cerebral cortex: Location and strength of an equivalent current dipole. Acta Neurol Scand 65:553-569.

Kiebel SJ, Holmes AP (2003): The general linear model. In: Frackowiak RSJ, Friston KJ, Frith C, Dolan R, Price CJ, Zeki S, Ashburner J, Penny WD, editors. Human Brain Function. San Diego: Academic Press. pp 725-760.

Gibson AP, Austin T, Everdell NL, Schweiger M, Arridge SR, Meek JH, Wyatt JS, Delpy DT, Hebden JC (2006): Three-dimensional whole-head optical tomography of passive motor evoked responses in the neonate. Neuroimage 30:521-528.

Heiskala J, Hiltunen P, Nissilä I (2009): Significance of background optical properties, time-resolved information and optode arrangement in diffuse optical imaging of term neonates. Phys Med Biol 54:535-554.

Hellige JB (1993): Hemispheric asymmetry: What's right and what's left. Cambridge: Harvard University Press. 412 p.

Hintz S, Benaron D, Siegel A, Zourabian A, Stevenson D, Boas D (2001): Bedside functional imaging of the premature infant brain during passive motor activation. J Perinat Med 29:335-343.
Homae F, Watanabe H, Nakano T, Asakawa K, Taga G (2006): The right hemisphere of sleeping infant perceives sentential prosody. Neurosci Res 54:276-280.

Huotilainen M (2006): Magnetoencephalography of the newborn brain. Semin Fetal Neonatal Med 11:437-443.

Isobe K, Kusaka T, Nagano K, Okubo K, Yasuda S, Kondo M, Itoh S, Onishi S (2001): Functional imaging of the brain in sedated newborn infants using near infrared topography during passive knee movement. Neurosci Lett 299:221224.

Karen T, Morren G, Haensse D, Bauschatz AS, Bucher HU, Wolf $M$ (2008): Hemodynamic response to visual stimulation in newborn infants using functional near-infrared spectroscopy. Hum Brain Mapp 29:453-460.

Kiguchi M, Ichikawa N, Atsumori H, Kawaguchi F, Sato H, Maki A, Koizumi H (2007): Comparison of light intensity on the brain surface due to laser exposure during optical topography and solar irradiation. J Biomed Opt 12:062108.

Kotilahti K, Nissilä I, Huotilainen M, Mäkelä R, Gavrielides N, Noponen T, Björkman P, Fellman V, Katila T (2005): Bilateral hemodynamic responses to auditory stimulation in newborn infants. Neuroreport 16:1373-1377.

Kusaka T, Kawada K, Okubo K, Nagano K, Namba M, Okada H, Imai T, Isobe K, Itoh S (2004): Noninvasive optical imaging in the visual cortex in young infants. Hum Brain Mapp 22:122132.

Mehler J, Dupoux E (1994): What Infants know. Cambridge, MA, USA: Basil Blackwell.

Minagawa-Kawai Y, Mori K, Naoi N, Kojima S (2007): Neural attunement processes in infants during the acquisition of a language-specific phonemic contrast. J Neurosci 27:315-321.

Nakasato N, Fujita S, Seki K, Kawamura T, Matani A, Tamura I, Fujiwara S, Yoshimoto T (1995): Functional localization of bilateral auditory cortices using an MRI-linked whole head magnetoencephalography (MEG) system. Electroencephalogr Clin Neurophysiol 94:183-190.

Nishida T, Kusaka T, Isobe K, Ijichi S, Okubo K, Iwase T, Kawada K, Namba M, Imai T, Itoh S (2008): Extrauterine environment affects the cortical responses to verbal stimulation in preterm infants. Neurosci Lett 443:23-26.

Nissilä I, Kotilahti K, Fallström K, Katila T (2002): Instrumentation for the accurate measurement of phase and amplitude in optical tomography. Rev Sci Instrum 73:3306-3312.

Nissilä I, Noponen T, Kotilahti K, Tarvainen T, Schweiger M, Lipiäinen L, Arridge S, Katila T (2005): Instrumentation and calibration methods for the multichannel measurement of phase and amplitude in optical tomography. Rev Sci Instrum 76: 044302.

Pena M, Maki A, Kovacic D, Dehaene-Lamberts G, Koizumi H, Bouquet F, Mehler J (2003): Sounds and silence: An optical topography study of language recognition at birth. Proc Natl Acad Sci USA 100:11702-11705.

Raichle ME, Mintun MA (2006): Brain work and brain imaging. Annu Rev Neurosci 29:449-476.

Sankoh AJ, Huque MF, Dubey SD (1997): Some comments on frequently used multiple endpoint adjustment methods in clinical trials. Stat Med 16:2529-2542.

Smith SM (2004): Overview of fMRI analysis. Br J Radiol 77:S167S175

Taga G, Asakawa K, Maki A, Konishi Y, Koizumi H (2003): Brain imaging in awake infants by near-infrared optical topography. Proc Natl Acad Sci USA 100:10722-10727. 
Taga G, Asakawa K (2007): Selectivity and localization of cortical response to auditory and visual stimulation in awake infants aged 2 to 4 months. Neuroimage 36:1246-1252.

Tarvainen T, Kolehmainen V, Vauhkonen M, Vanne A, Gibson AP, Schweiger M, Arridge SR, Kaipio JP (2005): Computational calibration method for optical tomography. Appl Opt 44:1879-1888.

Tervaniemi M, Hugdahl K (2003): Lateralization of auditory-cortex functions. Brain Res Rev 43:231-246.
Zaramella P, Freato F, Amigoni A, Salvadori S, Marangoni P, Suppjei A, Schiavo B, Chiandetti L (2001): Brain auditory activation measured by near-infrared spectroscopy (NIRS) in neonates. Pediatr Res 49:213-219.

Zeff BW, White BR, Dehghani H, Schlaggar BL, Culver JP (2007): Retinotopic mapping of adult human visual cortex with highdensity diffuse optical tomography. Proc Natl Acad Sci USA 104:12169-12174. 